\title{
ADDENDUM TO A PAPER OF CRAIG AND GOODMAN
}

\section{ARTHUR D. GORMAN}

Department of Mathematics

Lafayette College

Easton, Pennsylvania 18042, U.S.A.

(Received August 5, 1993)

\begin{abstract}
In [1], Craig and Goodman develop the geometrical optics solution of the linearized Korteweg-deVries equation away from caustic, or turning, points. Here we develop an analogous solution valid at caustic points.
\end{abstract}

KEY WORDS AND PHRASES. Korteweg-deVries equation, geometrical optics, Lagrange manifold, caustic points.

1991 AMS SUBJECT CLASSIFICATION CODES. 34E20, 35 Q53.

1. INTRODUCTION. In [1], Craig and Goodman consider the linearized Korteweg-deVries $(K d V)$ equation

$$
\partial_{t} u(x, t)=a(x, t) \partial_{x}^{3} u(x, t) .
$$

where $x$ represents space and $t$ represents time. Their analysis begins with a geometrical optics $(W K B)$ solution. They first rescale the independent variables to obtain

$$
\partial_{t} u(x, t)=\epsilon^{2} a(x, t) \partial_{x}^{3} u(x, t)
$$

where $\epsilon$ is a small parameter, then assume a solution of the form

$$
u(x, t)=A(x, t ; \epsilon) \exp \left[\frac{i}{\epsilon} S(x, t)\right]
$$

where $A(x, t ; \epsilon)=\sum_{j=0}^{\infty} \epsilon^{\jmath} A$, may be regarded as amplitudes and $S(x, t)$ as the phase. Then substituting (1.3) into (1.2) and regrouping, they develop an eikonal equation for the phase

$$
\partial_{t} S+a(x, t)\left(\partial_{x} S\right)^{3}=0
$$

a first order transport equation for $A_{0}$

$$
\partial_{t} S+3 a(x, t) \partial_{x}\left[\left(\partial_{x} S\right)^{2} A_{0}\right]=0
$$

and the Hamiltonian, $H(x, t, k, w)=w+a(x, t) k^{3}$, flow

$$
\begin{array}{ll}
\dot{x}=\partial_{k} H=3 a(x, t) k^{2} & \dot{k}=-\partial_{x} H=-\partial_{x}\left(a(x, t) k^{3}\right) \\
\dot{t}=\partial_{w} H=1 & \dot{w}=-\partial_{t} H=-\partial_{t}\left(a(x, t) k^{3}\right),
\end{array}
$$


where $\dot{w}=-\partial_{t} S$ and the dots indicate differentiation with respect to time. Near caustic curves this system is singular and the geometrical optics technique does not apply. An approach which does apply at caustics is the Lagrange manifold technique ([2], [3], [4]). Here we apply this technique at caustics associated with the linearized Korteweg-deVries equation.

\section{FORMALISM.}

Near caustics we assume an asymptotic solution of the form

$$
u(x, t)=\int A(x, k, t ; \epsilon) \exp \left[\frac{i}{\epsilon}(x k-S(k, t))\right] d k,
$$

where amplitude $A(x, k, t ; \epsilon)=\sum_{j=0}^{\infty} \epsilon^{\mathrm{j}} A$, and its derivatives are assumed bounded and $x k-S(k, t)=\phi(x, k, t)$ may be regarded as a phase. Then carrying the differentiation in (1.2) across the integral in (2.2) leads to

$$
\begin{aligned}
\int\left[\frac{i}{\epsilon} A\left(-\partial_{t} S+a(x, t) k^{3}\right)+\right. & \left(\partial_{t} A+3 a(x, t) k^{2} \partial_{x} A\right) \\
& \left.-i \epsilon\left(3 k a(x, t) \partial_{x}^{2} A\right)-\epsilon^{2} a(x, t) \partial_{x}^{3} A\right] \exp \left[\frac{i}{\epsilon} \phi\right] d k=0(\epsilon) .
\end{aligned}
$$

Here, the coefficient of the $\frac{1}{\epsilon}$ term is the Hamiltonian. On the caustic, the integral is evaluated using the stationary phase condition $\left[\partial_{k} \phi=0\right]$, which obtains the Lagrange manifold

$$
x=\partial_{k} S(k, t)
$$

and turns the Hamiltonian into an eikonal equation, cf. (1.4),

$$
-\partial_{t} S+a(x, t) k^{3}=0
$$

We obtain the phase from the Hamiltonian flow, (1.5). First these equations are solved to obtain

$$
\begin{array}{ll}
x=x(\mu, \sigma) & k=k(\mu, \sigma) \\
t=t(\mu, \sigma) & w=w(\mu, \sigma),
\end{array}
$$

using $\mu$ as an arbitrary ray-path parameter and $\sigma$ as an initial condition. Then the inversion of the wave vector $(k)$ and time transformations, followed by substitution into the coordinate space map, determines the Lagrange manifold explicitly,

$$
x=x(\mu(t, k), \sigma(t, k))=\partial_{k} S(k, t) .
$$

An integration along the trajectories obtains

$$
S(k, t)=\int_{k_{0}}^{k} x d k
$$

and hence the phase

$$
\phi(x, k, t)=x k-S(k, t)
$$

We obtain a transport equation for the amplitudes by first Taylor-expanding the Hamiltonian near the Lagrange manifold to obtain

$$
\begin{aligned}
A(x, k, t ; \epsilon)\left(-\partial_{t} S+a(x, t) k^{3}\right)= & A\left(\partial_{k} S, k, t ; \epsilon\right)\left(-\partial_{t} S+a\left(\partial_{k} S, t\right) k^{3}\right) \\
& +\left(x-\partial_{k} S\right) D=\left(x-\partial_{k} S\right) D
\end{aligned}
$$


where

$$
D=\int_{0}^{1} \partial_{x} H\left(\gamma\left(x-\partial_{k} S\right)+\partial_{k} S, k, t\right) d \gamma
$$

with $H$ the Hamiltonian, i.e., $H=\partial_{t} S+a(x, t) k^{3}$. Next substituting into (2.2) leads to

$$
\begin{aligned}
\int\left[\left(-\partial_{k} A\right) D\right. & +\left(-\partial_{k} D\right) A+\partial_{t} A+3 a(x, t) k^{2} \partial_{x}^{2} A \\
& \left.-i \epsilon a(x, t)\left(3 k \partial_{x}^{2} A\right)-\epsilon^{2} a(x, t) \partial_{x}^{3} A\right] \exp \left[\frac{i}{\epsilon} \phi\right] d k=0(\epsilon) .
\end{aligned}
$$

Finally, introducing the non-Hamiltonian flow

$$
\dot{x}=3 a(x, t) k^{2} \partial_{x}^{2} A \quad k=-D \quad \dot{t}=1
$$

and requiring that

$$
\begin{aligned}
\left(-\partial_{k} A\right) D+ & \left(-\partial_{k} D\right) a+\partial_{t} A+3 a(x, t) k^{2} \partial_{x}^{2} A \\
& -i \epsilon a(x, t)\left(3 k \partial_{x}^{2} A\right)-\epsilon^{2} a(x, t) \partial_{x}^{3} A=0
\end{aligned}
$$

in a neighborhood of the Lagrange manifold determines the transport equation

$$
\frac{d A}{d t}-A, \partial_{k} D-3 k a(x, t) \partial_{x}^{2} A,-a(x, t) \partial_{x}^{3} A_{\jmath-2}=0
$$

for the evolution of the amplitudes $A$,

The asymptotic evaluation of the field integrals at the caustic points has been detailed elsewhere ([2], [3], [4]). For brevity, we do not repeat the procedure here.

\section{REFERENCES}

1. CRAIG, W. and GOODMAN, J., Linear dispersive equations of Airy type, J. Diff. Eqns. 87 (1990), 38-61.

2. ARI, N. and GORMAN, A.D., Time-evolution of a caustic, Internat. J. Math. and Math. Sci. 11 (1988), 805-810.

3. GORMAN, A.D., On caustic associated with hyperbolic systems, Quart. Appl. Math. XIIX (1991), 773-780.

4. GORMAN, A.D., On the asymptotic series solution of some higher order linear differential equations at turning points, Internat. J. Math. and Math. Sci. 7 (1984), 541-548. 


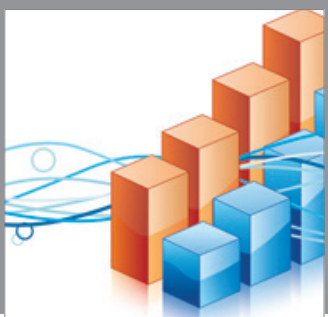

Advances in

Operations Research

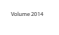

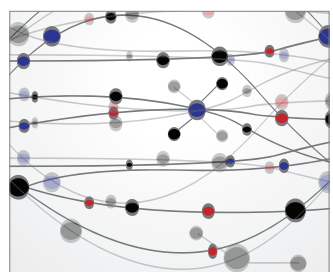

\section{The Scientific} World Journal
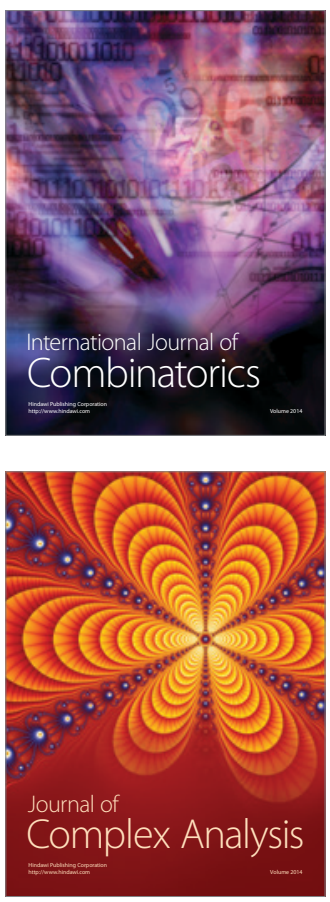

International Journal of

Mathematics and

Mathematical

Sciences
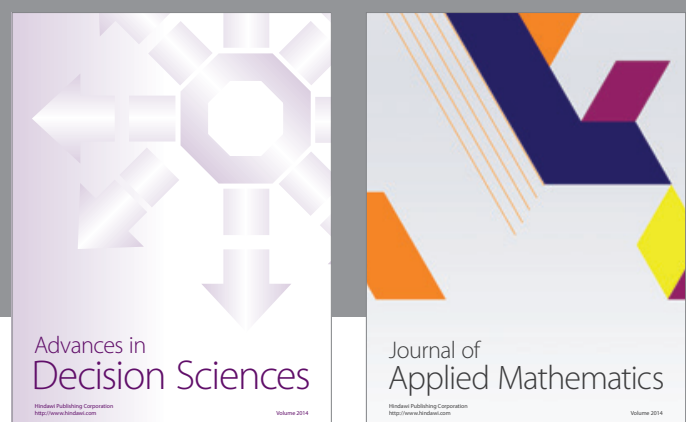

Journal of

Applied Mathematics
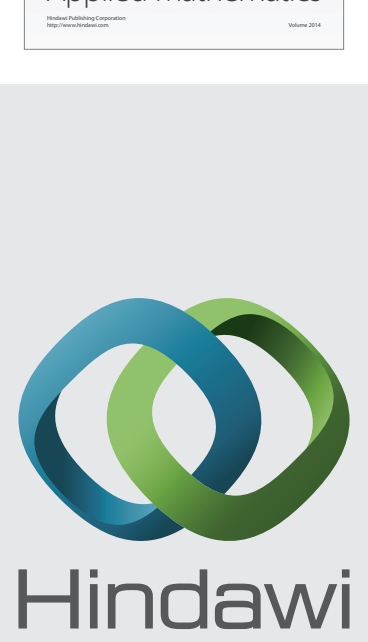

Submit your manuscripts at http://www.hindawi.com
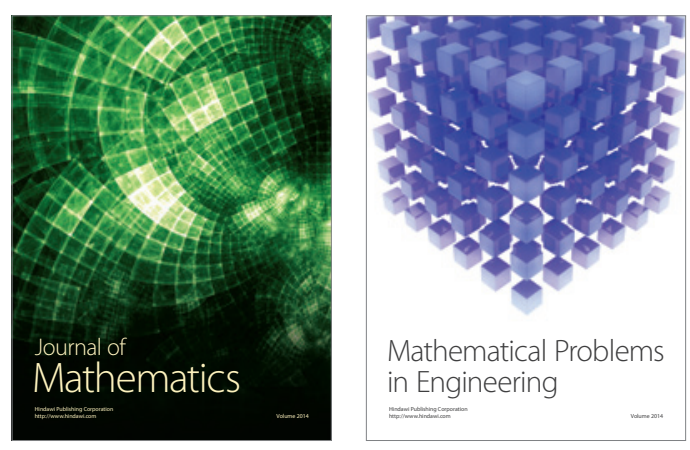

Mathematical Problems in Engineering
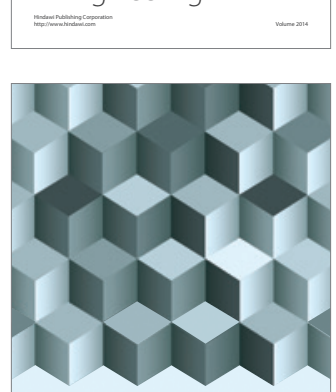

Journal of

Function Spaces
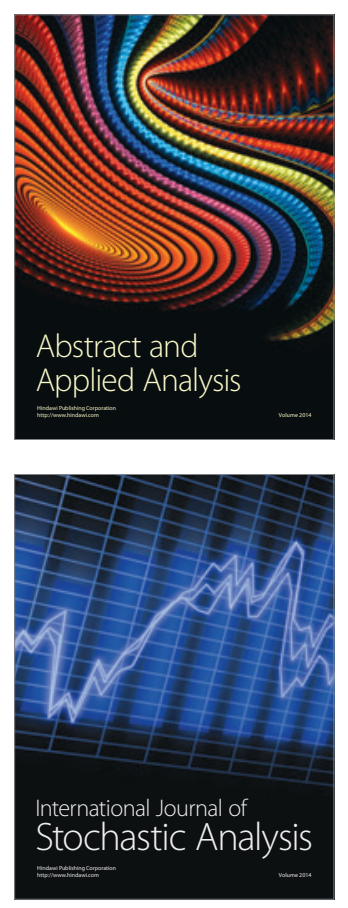

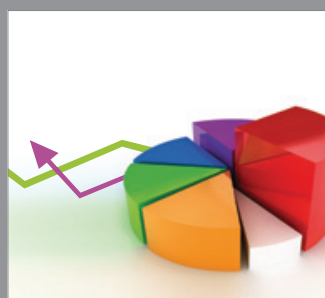

ournal of

Probability and Statistics

Promensencen
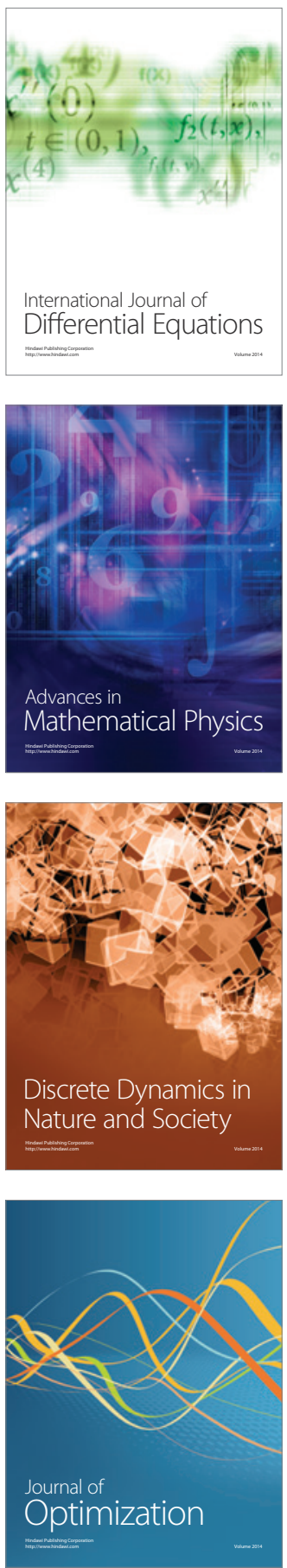